\title{
Exosomes derived from microRNA-584 transfected mesenchymal stem cells: novel alternative therapeutic vehicles for cancer therapy
}

\author{
Ran Kim ${ }^{1}$, Seokyeon Lee ${ }^{1}$, Jihyun Lee ${ }^{1}$, Minji Kim ${ }^{1}$, Won Jung Kim ${ }^{1}$, Hee Won Lee ${ }^{1}$, Min Young Lee ${ }^{2}$, Jongmin Kim ${ }^{3}$ E $^{2}$ \\ Woochul Chang ${ }^{1, *}$ \\ ${ }^{1}$ Department of Biology Education, College of Education, Pusan National University, Busan 46241, ${ }^{2}$ Department of Molecular Physiology, \\ College of Pharmacy, Kyungpook National University, Daegu 41566, ${ }^{3}$ Department of Life Systems, Sookmyung Women's University, Seoul \\ 04310, Korea
}

\begin{abstract}
Exosomes are small membranous vesicles which contain abundant RNA molecules, and are transferred from releasing cells to uptaking cells. MicroRNA (miRNA) is one of the transferred molecules affecting the adopted cells, including glioma cells. We hypothesized that mesenchymal stem cells (MSCs) can secrete exosomes loading miRNA and have important effects on the progress of gliomas. To determine these effects by treating exosomal miRNA in culture media of miRNA mimic transfected MSCs, we assessed the in vitro cell proliferation and invasion capabilities, and the expression level of relative proteins associated with cell apoptosis, growth and migration. For animal studies, the mice injected with U87 cells were exposed to exosomes derived from miRNA-584-5p transfected MSCs, to confirm the influence of exosomal miRNA on the progress of glioma. Based on our results, we propose a new targeted cancer therapy wherein exosomes derived from miRNA transfected MSCs could be used to modulate tumor progress as the anticancer vehicles. [BMB Reports 2018; 51(8): 406-411]
\end{abstract}

\section{INTRODUCTION}

Malignant gliomas are the most common primary brain tumors affecting the central nervous system, with accompanying high morbidity and mortality $(1,2)$. Despite current advances in surgical resection techniques, radiation therapy and chemotherapy, the prognosis of glioma remains extremely poor $(3,4$, 5). Limitations of these therapies include incomplete removal

*Corresponding author. Tel: +82-51-510-3124; Fax: +82-51-5148576; E-mail: wchang1975@pusan.ac.kr

https://doi.org/10.5483/BMBRep.2018.51.8.105

Received 8 May 2018, Revised 28 May 2018, Accepted 27 June 2018

Keywords: Cancer therapy, Exosomes, Glioma, Mesenchymal stem cells, MicroRNA of gliomas during surgery, impact of irradiation on surrounding normal brain tissues as well as the target tissues, and difficult for infiltration of chemicals to cross the blood brain barriers (2, 6). Based on these limitations, some researchers turned their attention to alternate candidates for cancer management.

Mesenchymal stem cells (MSCs) are recently highlighted as a new approach to overcome the demerits of previous gliomas therapies $(2,7)$. In recent years, cancer related studies using MSCs have got interested in tropism for tumor sites (8). The tropism of MSCs is based on their inherent homing ability towards damaged or injured tissues in response to released substances and factors from the tissues $(2,9)$. After reaching the tumor site, MSCs show anti-tumor properties, such as decreasing proliferation and increasing apoptosis of cancer cells $(7,10,11)$. MSCs secrete numerous bioactive molecules including exosomes that contribute to establishing immunomodulatory functions, and remodeling a suitable microenvironment for preparation of tissue regeneration (10). Exosomes are small membranous vesicles secreted from a variety of cell types (12). These vesicles contain microRNAs (miRNAs) and can transfer from cell to cell and contributes to cell-to-cell communication, modulation of cellular activities of target cells $(13,14,15)$. Some studies have reported that the intercellular transport of miRNAs occurs through exosomes $(16,17)$. MiRNAs, as gene regulators, modulate diverse biological processes, during development, such as cell proliferation, differentiation, and apoptosis (18). MiRNA-584, a major tumor suppressive biomarker in various cancers, is known to inhibit tumor cell growth, metastasis, and invasion through decrease of oncogenes, and induce cell apoptosis by blocking the expression and activity of relative proteins (19-22). It targets the expression of CYP2J2 which is associated with the pathogenesis and progression of metastatic cancers.

In this study, we hypothesized that exosome derived from MSCs may load miRNA-584 and exosomal miRNA-584 regulate the activities of malignant glioma cells. We demonstrated the effects of exosomal miRNA-584 derived from transfected MSCs on cell proliferation, migration and

ISSN: 1976-670X (electronic edition)

Copyright (c) 2018 by the The Korean Society for Biochemistry and Molecular Biology

(c) This is an open-access article distributed under the terms of the Creative Commons Attribution Non-Commercial License (http://creativecommons.org/licenses/by-nc/4.0) which permits unrestricted non-commercial use, distribution, and reproduction in any medium, provided the original work is properly cited. 
invasion of U87 in vitro, and alleviation of tumor progression in vivo. Summarizing our results, we propose that utilization of MSCs derived exosomes as vehicles of therapeutic miRNAs is a new cell-based strategy for tumor therapies.

\section{RESULTS}

\section{Effect of miRNA-584 for CYP2J2 expression of U87 cells}

To investigate the regulatory effects of miRNA-584 for expression of CYP2J2 as a tumor biomarker, we performed of miRNA-584-5p transfection and RT-PCR with transfected U87. Based on Target Scan, miRNA-584 was selected as a regulator of CYP2J2. We expected that the binding site of miRNA-584 is located in CYP2J2 gene, thereby inhibiting the gene expression. In Fig. 1A, CYP2J2 expression was substantially reduced in miRNA-584-5p transfected U87. Fig. 1B shows the sequence of miRNA-584 and its target gene, CYP2J2. To determine whether miRNA-584 was bound with 3 '-UTR of CYP2J2, a control or pmirGLO-CYP2J2 vector was co-transfected with miRNA-584-5p or a negative control in U87. In

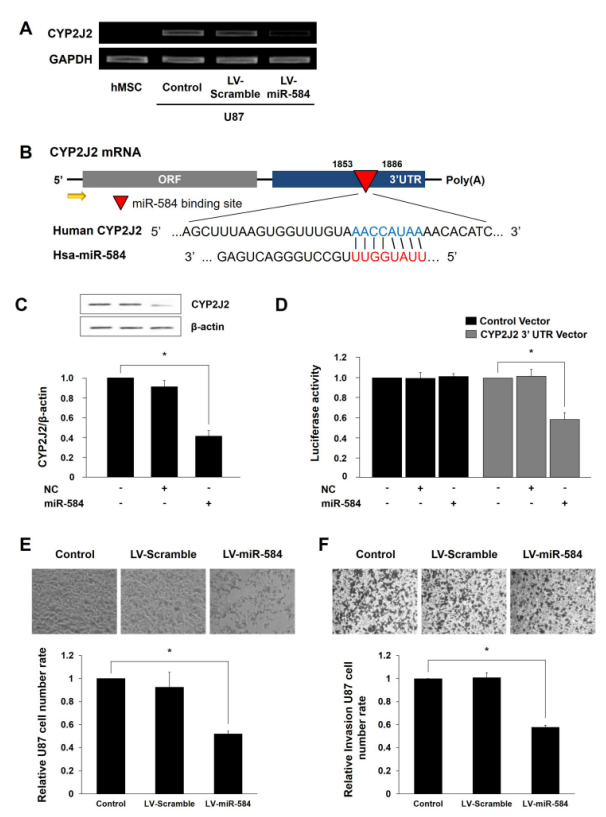

Fig. 1. Effect of miRNA-584 for CYP2J2 expression and pathophysiological changes in miRNA-584 transfected U87 cells. (A) The data of RT-PCR show miRNA-584 transfection substantially reduced CYP2J2 expression of U87. (B) The sequence of miRNA-584 shows it regulates the expression of CYP2J2. (C) Transfection of miRNA-584 decreased CYP2J2 expression. (D) The data of luciferase assay indicate decreased CYP2J2 expression by miRNA-584 transfection. (E, F) Proliferation and migratory ability of miRNA-584 transfected U87 was analyzed with light microscopic photographs and statistical analysis. Experiments were repeated three times. LV-Scramble, lentivirus-pre-miRNA-scramble sequence; LV-miR-584, lentivirus-pre-miRNA-584. Columns, mean; bars, SE. $(* P<0.05)$.
miRNA-584-5p transfected U87, there was a decrease in CYP2J2 expression, and targeting 3'-UTR of CYP2J2 resulted in decreased luciferase activity (Fig. 1C, D). Taken together, these findings indicate that miRNA-584 suppresses the expression of CYP2J2, which could inhibit the activity of U87.

\section{Pathophysiological changes of U87 cells by transfected miRNA-584}

We evaluated whether exosomal miRNA-584 of transfected MSCs affected cell proliferation and invasion abilities of U87. Because tumor pathophysiology plays a central role in growth, invasion and metastasis of cancer cells (23). Fig. 1E indicates cell proliferation was significantly decreased in miRNA-584-5p transfected U87. Especially, the number of transfected U87 reduced almost 2.0-fold than observed in control group. In addition, Fig. $1 \mathrm{~F}$ shows the migratory ability of transfected U87 was inhibited 1.7-fold as compared to control. Based on these results, we deduce that increased miRNA-584 in U87 diminished the tumor cell proliferation and invasion abilities by targeting CYP2J2.

\section{Efficacies of exosomal miRNA-584 derived from MSCs for U87 cells}

After confirming that culture media (CM) of miRNA-584 transfected MSCs contained exosomes using exosome and intracellular markers (Fig. 2A), we next examined whether extracellular miRNAs are located within exosomes secreted from transfected MSCs. MiRNA-584 was present in high concentrations in extracellular vesicles (EV)-584 treated group,

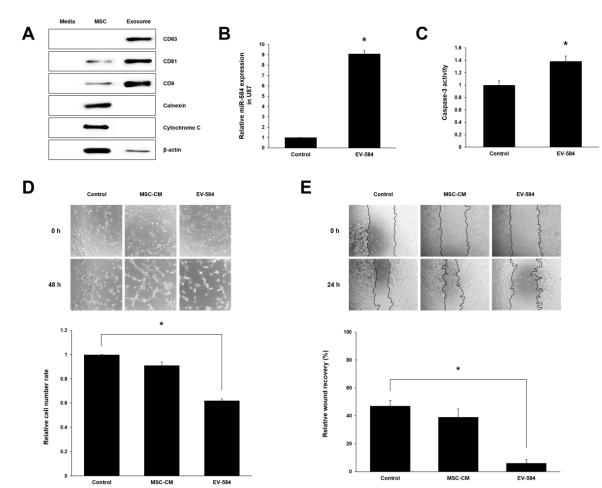

Fig. 2. Efficacies of exosomal miRNA-584 derived from transfected MSCs for U87 cells. (A) Expression of exosomes markers were identified in exosomes isolated from CM of miRNA-584 transfected MSCs by western blot. (B) The qPCR data show miRNA-584 were high in U87 treated exosomes derived from transfected MSCs. (C) Caspase-3 activity was increased in co-cultured U87 with CM of transfected MSCs. (D, E) Proliferation assay and migration assay of U87 treated with exosomal miRNA-584 derived from transfected MSCs. Experiments were repeated three times. MSC-CM, culture media of MSCs; EV-584, extracellular vesicles (exosomal miRNA-584) derived from transfected MSCs. Columns, mean; bars, SE. $\left({ }^{*} \mathrm{P}<0.05\right)$. 
as compared to those of control group where miRNA-584 was rarely detected in U87 (Fig. 2B). This indicates that U87 treated exosomal miRNA-584 from transfected MSCs generated more miRNA-584 than control. Because miRNA-584 had cytotoxic effects and apoptotic-inducing abilities in several types of human cancer (19-21), we resorted to evaluating caspase-3 activity of miRNA-584-5p transfected MSCs derived exosomes treated U87. The caspase-3 activity of U87 was enhanced by treatment of exosomal miRNA-584 from transfected MSCs as compared to control (Fig. 2C). This indicates that U87 underwent apoptosis in response to exosomal miRNA-584 derived from transfected MSCs. In Fig. 2D, U87 treated with exosomes from miRNA-584-5p transfected MSCs significantly inhibited cell proliferation, which is similar to directly miRNA-584-5p transfected U87. In addition, we also examined whether exosomal miRNA-584 from transfected MSCs affected the migration ability of U87. The in vitro wound healing assay showed that the ability of U87 was considerably attenuated by treatment of transfected MSCs derived exosomes (Fig. 2E). These results indicate that exosomal miRNA-584 present in the CM of transfected MSCs could be delivered to $U 87$, resulting in a reduction in cell proliferation and migration capabilities of glioma cells.

\section{Expression of related genes of U87 cells through treating miRNA-584 transfected MSCs derived exosomes}

Invasion is a major property of malignant tumors, and is associated with proteolytic degradation of extracellular matrix

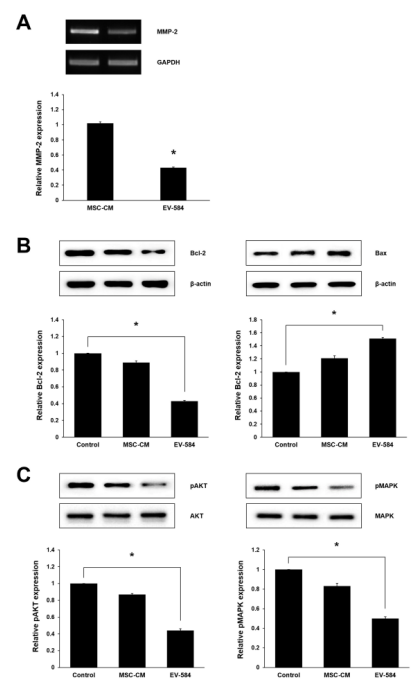

Fig. 3. Expression of related genes of U87 cells by treatment of exosomal miRNA-584 of transfected MSCs. (A) The data of RT-PCR indicate MMP-2 mRNA expression was significantly reduced in group treated with exosomes derived from miRNA-584 transfected MSCs. (B, C) Expression levels of related genes of U87 viability, growth and invasion were confirmed by western blot. Experiments were repeated three times. Columns, mean; bars, SE. $\left({ }^{*} P<0.05\right)$.
(ECM) components including matrix metalloproteinases (MMPs) (13). MMP-2 plays an important role in cancer progression, and is highly expressed in gliomas as compared with normal tissue. We investigated whether exosomal miRNA-584 from transfected MSCs affected MMP-2 expression in U87. Our results show that the mRNA expression of MMP-2 in U87 treated with exosomes derived from transfected MSCs was significantly reduced by 2.5 -fold as compared to the control (Fig. 3A). We further evaluated the expression level of relative proteins associated with cell apoptosis, growth and migration. U87 exposed to exosomes containing miRNA-584 had decreased expression levels of the anti-apoptotic protein (Bcl-2) and increased levels of pro-apoptotic protein (Bax) (Fig. 3B). Previous studies have determined that AKT is associated with cancer growth and migration through inhibiting tumor cell apoptosis, and MAPK can be activated in response to a diverse range of extracellular stimuli including mitogen, growth factors, and cytokines (13, 24, 25). We therefore examined whether transfected MSCs derived exosomal miRNA-584 affected the phosphorylation of AKT and MAPK. Our results indicate that treatment of the exosomal miRNA-584 on U87 significantly reduced the levels of phosphorylated AKT and MAPK (Fig. 3C).

\section{Suppression of tumor progress by treatment of exosomes derived from miRNA-584 transfected MSCs in vivo} To confirm the effect of exosomes derived from miRNA-584-5p transfected MSCs on glioma development, we

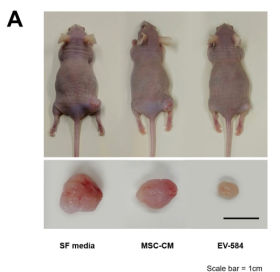

B

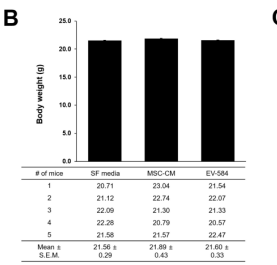

C

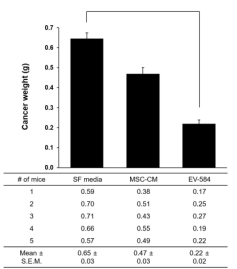

Fig. 4. Suppression of glioma development after exposure to exosomes derived from miRNA-584 transfected MSCs. (A) Tumor sizes in each group were analyzed. (B) Body weights showed no significant difference between groups. (C) Statistical analysis revealed that cancer weight in group exposed to exosomal miRNA-584 derived from transfected MSCs was considerably decreased. All data were analyzed after each treatment on 5 days a week for 30 days. Experiments were repeated three times. SF media, serum-free media. Scale bar, $1 \mathrm{~cm}$. Columns, mean; bars, SE. $\left({ }^{*} P<0.05\right)$ 
used the in vivo $U 87$ xenograft nude mouse model. Mice were treated with serum-free (SF) media, MSCs-CM and exosomes containing miRNA-584 derived from transfected MSCs. Aggressive tumor growth was observed in SF media treatment groups, while suppressive tumor growth was identified in exosomes of transfected MSCs treatment group (Fig. 4A). We examined the change in body and tumor mass weights of all three groups. The tumor mass weights in the exosomes containing miRNA-584 treatment group considerably decreased when compared with other groups (Fig. 4B). However, the exosomal miRNA-584 treatment group did not show significant difference in body weights in comparison to SF media and MSCs-CM treatment groups (Fig. 4C).

\section{DISCUSSION}

In the present study, we demonstrated that miRNA mimic packaged into exosomes of MSCs are integrated in glioma cells and are attributable to activities of gliomas. MSCs as an alternative material for glioma treatment have gained attention due to their ability of tropism to track tumor cells and delivery of therapeutic drugs or genes exerting antitumor effects (1, 26-29). Especially, MSCs release large amounts of exosomes that contribute to genetic communication, and supply the exogenous miRNA mimics on glioma cells, providing an efficient route of therapeutic miRNA delivery $(30,31)$. MiRNA-584 acts as a tumor suppressor in some cancers and suppresses the activity of glioma cells by binding to the 3'-UTR of CYP2J2 $(21,22)$. On the basis of these factors, we confirmed that CYP2J2 expression in miRNA-584-5p transfected U87 was significantly decreased, thereby resulting in reduced proliferation and invasive ability of the glioma cells (Fig. 1). We hypothesized that exosomal miRNA-584 were transferred from MSCs to U87, and affected the activities of U87 in vitro and the tumor progress in vivo. To analyze the effects of MSCs derived exosomal miRNA-584 on glioma, we confirmed the existence of exosomes and the quantity of exosomal miRNA-584 in CM of transfected MSCs using exosome markers (CD9, CD81 and CD63) and intracellular markers (calnexin and cytochrome C). In addition, U87 treated with exosomes containing miRNA-584 showed significantly reduced cell proliferation and migration in vitro (Fig. 2). Proteolytic degradation of ECM components is also necessary for invasion of malignant tumors, and MMPs are major regulators of ECM proteins (13). Our studies revealed that U87 treated with exosomal miRNA-584 of transfected MSCs have reduced expression of MMP-2. This result suggests that decrease of MMP-2 expression through treatment of transfected MSCs derived exosomal miRNA-584 could suppress glioma metastasis. We further confirmed that miRNA-584 in exosomes derived from transfected MSCs were associated with cell apoptosis, by assessing the related protein expressions such as $\mathrm{Bcl}-2$ and Bax. We observed decreased expression of $\mathrm{BCl}-2$, and an increased expression of $\mathrm{Bax}$, in
U87 treated with exosomal miRNA-584. We also investigated if miRNA-584 in exosomes derived transfected MSCs affected the signaling pathway in U87. CYP2J2 promotes tumor cell proliferation and protects carcinoma cells from apoptosis through activation of both AKT and MAPK pathways $(18,25)$. We therefore investigated phosphorylated AKT and MAPK expression, and both levels showed a decline in the MSCs derived exosomal miRNA-584 treated U87 in vitro (Fig. 3). Furthermore, we demonstrated the effects of exosomal miRNA-584 in CM of MSCs on progression of glioma with U87 injected in athymic NCr-nu/nu mice. Our in vivo data revealed attenuation of the malignant gliomas after exposure to the exosomes derived from miRNA-584 transfected MSCs; the treatment had no effect on body weight of the animals (Fig. 4).

This study ascertained whether transferred miRNA through exosomes from MSCs affects the activities of glioma cells and the tumor progression. After careful deliberation of our data, we propose that release of specific therapeutic miRNA through exosomes of MSCs could be considered as an alternative strategy for treatment of various cancers, including malignant gliomas.

\section{MATERIALS AND METHODS}

\section{Cell culture}

U87 human glioma cells were obtained from the American Type Culture Collection (ATCC, VA, USA), and human MSCs (hMSCs; Cat no: PT-2501) were purchased from Lonza (MD, USA). U87 and hMSCs were maintained routinely in Dulbecco's Modified Eagle's Media (DMEM; HyClone, UT, USA) supplemented with $10 \%$ Fetal Bovine Serum (FBS; HyClone) and $1 \%$ Penicillin-Streptomycin antibiotic solution (HyClone).

\section{Transfection}

Transfection was conducted with using lentivirus-mediated transfer of pre-miRNA (LV-miR) precursor molecule (Macrogen, Seoul, Korea). Cells $\left(7 \times 10^{5}\right)$ were plated and transfected with $100 \mathrm{nM}$ LV-miR-584-5p or LV-miR of scrambled sequence at a multiplicity of injection (MOI) of 5 in the presence of $6 \mu \mathrm{g} / \mathrm{ml}$ polybrene (Sigma-Aldrich, MO, USA).

\section{Total RNA isolation and cDNA synthesis}

Total RNA was prepared using a Hybrid-R RNA purification kit (GeneAll, Seoul, Korea) and converted to cDNA using the cDNA synthesis kit (Thermo Scientific, Vilnius, Lithuania). Amplification using RT-PCR kit (Solgent, Daejeon, Korea) was performed in a DNA thermal cycler under the appropriate conditions. The PCR products were electrophoresed on a $1.0 \%$ agarose gel.

\section{Luciferase assay}

We designed a molecular beacon (MB; Bionics, Seoul, Korea) for miRNA-584-5p that could form a partially double stranded 
oligonucleotide. The predicted target of miRNA-584 was identified using Target Scan (www.targetscan.org). The corresponding genes were cloned into the pmirGLO vector. hMSCs $\left(3 \times 10^{4}\right)$ were plated in 24-well plates. After $48 \mathrm{~h}$, the pmirGLO vector containing the CYP2J2 binding site for miRNA-584 was co-transfected with either miRNA-584 or the negative control, using Lipofectamine 2000 (Invitrogen, CA, USA). Luciferase activity was measured using the Dual Luciferase assay (Promega, WI, USA).

\section{Preparation of exosomes containing miRNA-584}

MiRNA-584-5p or scrambled RNA oligomers were transfected into hMSCs $\left(7 \times 10^{5}\right)$. After $48 \mathrm{~h}$, the supernatant was collected and centrifuged at 2,000 $\times \mathrm{g}$ for $10 \mathrm{~min}$ for removal of cellular debris. For collection of exosome fraction, the supernatant was transferred to new tubes and centrifuged at least at $100,000 \times \mathrm{g}$ for $70 \mathrm{~min}$. And the pellet was resuspended in $1 \mathrm{ml}$ phosphate-buffered saline (PBS; HyClone) and then washed with PBS. The final centrifugation was performed at $100,000 \times \mathrm{g}$ for $1 \mathrm{~h}$.

\section{Cell proliferation assay}

U87 $\left(5 \times 10^{4}\right)$ were plated in 24-well plate. After $3 \mathrm{~h}$, the medium was replaced with $\mathrm{CM}$ containing exosomes from miRNA-584 transfected MSCs. U87 were observed using a light microscope (Nikon, Tokyo, Japan) and counted.

\section{Transwell invasion assay}

Invasion was evaluated by performing the modified Boyden chamber assay. U87 $\left(3 \times 10^{4}\right)$ were seeded into the upper chamber, and the lower chamber was filled with CM supplemented with $20 \%$ FBS as the chemoattractant. After $24 \mathrm{~h}$, U87 that had not migrated through the upper side of filters were scraped off with a cotton wool swab. The filters were washed in PBS, fixed with $4 \%$ formaldehyde (Sigma-Aldrich, MO, USA), and stained with $0.1 \%$ Coomassie blue (Santa Cruz Biotechnology, CA, USA).

\section{Western blot}

Cells were collected in lysis buffer, and equal quantities of proteins were subjected to $10 \%$ sodium dodecyl sulfate-polyacrylamide gel electrophoresis. Next, the proteins were transferred to polyvinylidene difluoride membranes (Millipore, MA, USA). The membranes were blocked with Tris-buffered saline-Tween 20 (TBS-T, $0.05 \%$ Tween 20) containing $10 \%$ skim milk, following which they were exposed to the primary antibodies overnight. Membranes were washed with TBS-T and then incubated with a horseradish peroxidase conjugated secondary antibody (Thermo Scientific). Immunoreactive proteins were detected using the WesternBright ECL HRP substrate (Advansta, CA, USA).
Real-time polymerase chain reaction (PCR) analysis Total RNA was extracted using an RNeasy mini kit (Qiagen, CA, USA), and reverse transcription was performed using an Omniscript RT kit (Qiagen) with total RNA and oligo(dT) primer (Invirogen). Total synthesized cDNA was re-suspended in nuclease-free water and used for qPCR. The relative expression of each target gene was calculated using the threshold cycle $(\Delta \mathrm{Ct})$ method. $\Delta \mathrm{Ct}$ was normalized to the cycle number of GAPDH.

\section{Caspase-3 activity assay}

Following co-culture with CM of miRNA-584 transfected MSCs for 24 h, U87 were lysed in CelLytic M Cell Lysis reagent (Sigma-Aldrich), and protein concentration was determined by the Bradford assay. Subsequently, caspase-3 activity was confirmed using a CaspACE Assay System, according to the manufacturer's instructions (Promega).

\section{Wound healing assay}

U87 $\left(1 \times 10^{5}\right)$ were seeded in 24-well plate. After $24 \mathrm{~h}$, a scratch was created in the monolayer cell surface using a sterile pipette tip. The wells were subsequently washed with SF media to remove floating cells, and then incubated with SF media or supernatant containing exosomes. Images were taken of each well using the Meta-Morph imaging software version 7.5 (Molecular Devices, CA, USA).

\section{In vivo tumor xenograft study}

U87 $\left(2.5 \times 10^{6}\right)$ were injected subcutaneously into the right flank of male athymic NCr-nu/nu mice (6-weeks). After 1 week, the mice were randomly divided into 3 groups: treatment groups of SF media, MSCs-CM and exosomes derived from miRNA-584-5p transfected MSCs. All treatment was administered 5 days a week for 30 days. Animals were handled according to the animal welfare guidelines issued by the Korean National Institute of Health and the Korean Academy of Medical Sciences for the care and use of laboratory animals.

\section{Statistical analysis}

All data were expressed as the mean \pm SEM. Comparisons between more than two groups were performed by one-way ANOVA using Bonferroni's correction. A P value of $<0.05$ was considered statistically significant.

\section{ACKNOWLEDGEMENTS}

This research was supported by a 2-Year Research Grant of Pusan National University.

\section{CONFLICTS OF INTEREST}

The authors have no conflicting interests. 


\section{REFERENCES}

1. Xu F, Shi J, Yu B, Ni W, Wu X and Gu Z (2010) Chemokines mediate mesenchymal stem cells migration toward gliomas in vitro. Oncol Rep 23, 1561-1567

2. Yang C, Lei D, Ouyang W et al (2014) Conditioned media from human adipose tissue-derived mesenchymal stem cells and umbilical cord-derived mesenchymal stem cells efficiently induced the apoptosis and differentiation in human glioma cell lines in vitro. Biomed Res Int 2014, 109389

3. Brandes AA, Tosoni A, Franceschi E, Reni M, Gatta G and Vecht C (2008) Glioblastoma in adults. Crit Rev Oncol Hematol 67, 139-152

4. He Y, Zhao C, Liu Y et al (2016) miR-124 functions as a tumor suppressor via targeting hCLOCK1 in glioblastoma. Mol Neurobiol [Epub ahead of print]

5. Liao A, Shi R, Jiang $Y$ et al (2016) SDF-1/CXCR4 axis regulates cell cycle progression and epithelialmesenchymal transition via up-regulation of surviving in glioblastoma. Mol Neurobiol 53, 210-215

6. Sai K, Yang QY, Shen D and Chen ZP (2013) Chemotherapy for gliomas in mainland China: An overview. Oncol Lett 5, 1448-1452

7. Ho IA, Toh HC, Ng WH et al (2013) Human bone marrow-derived mesenchymal stem cells suppress human glioma growth through inhibition of angiogenesis. Stem Cells 31, 146-155

8. Reagan MR and Kaplan DL (2011) Concise review: mesenchymal stem cell tumor-homing: detection methods in disease model systems. Stem Cells 29, 920-927

9. Adjei IM and Blanka S (2015) Modulation of the tumor microenvironment for cancer treatment: a biomaterials approach. J Funct Biomater 6, 81-103

10. Mok PL, Leong CF and Cheong SK (2013) Cellular mechanisms of emerging applications of mesenchymal stem cells. Malays J Pathol 35, 17-32

11. Khakoo AY, Pati S, Anderson SA et al (2006) Human mesenchymal stem cells exert potent antitumorigenic effects in a model of Kaposi's sarcoma. J Exp Med 203, 1235-1247

12. Mathivanan S, Ji H and Simpson RJ (2010) Exosomes: extracellular organelles important in intercellular communication. J Proteomics 73, 1907-1920

13. Yan H, Wang W, Dou C, Tian F and Qi S (2015) Securin promotes migration and invasion via matrix metalloproteinases in gioma cells. Oncol Lett 9, 2895-2901

14. Valadi H, Ekstrom K, Bossios A, Sjostrand M, Lee JJ and Lotvall JO (2007) Exosome-mediated transfer of mRNAs and microRNAs is a novel mechanism of genetic exchange between cells. Nat Cell Biol 9, 654-659

15. Soung YH, Nguyen T, Cao H, Lee J and Chung J (2016) Emerging roles of exosomes in cancer invasion and metastasis. BMB Rep 49, 18-25

16. Katakowski M, Buller B, Zheng X et al (2013) Exosomes from marrow stromal cells expressing miR-146b inhibit glioma growth. Cancer Lett 335, 201-204

17. Ono M, Kosaka N, Tominage $N$ et al (2014) Exosomes from bone marrow mesenchymal stem cells contain a microRNA that promotes dormancy in metastatic breast cancer cells. Sci Signal 7, ra63

18. Chen F, Chen C, Yang S et al (2012) Let-7b inhibits human cancer phenotype by targeting cytochrome P450 epoxygenase 2J2. PLoS One 7, e39197

19. Fils-Aime N, Dai M, Guo J et al (2013) MicroRNA-584 and the protein phosphatase and actin regulator 1 (PHACTR1), a new signaling route through which transforming growth factor- $\beta$ Mediates the migration and actin dynamics of breast cancer cells. J Biol Chem 288, 11807-11823

20. Ueno K, Hirata H, Shahryari V et al (2011) Tumour suppressor microRNA-584 directly targets oncogene Rock-1 and decreases invasion. Br J cancer 104, 308-315

21. Xiang X, Mei H, Qu H et al (2015) MiRNA-584-5p exerts tumor suppressive functions in human neuroblastoma through repressing transcription of matrix metallo proteinase 14. Biochim Biophys Acta 1852, 1743-1754

22. Wang XP, Deng XL and Li LY (2014) MicroRNA-584 functions as a tumor suppressor and targets PTTG1IP in glioma. Int J Clin Exp Pathol 7, 8573-8582

23. Jiang JG, Ning YG, Chen C et al (2007) Cytochrome P450 epoxygenase promotes human cancer metastasis. Cancer Res 67, 6665-6674

24. You D, Jung SP, Jeong Y, Bae SY, Lee JE and Kim S (2017) Fibronectin expression is upregulated by PI-3K/Akt activation in tamoxifen-resistant breast cancer cells. BMB Rep 50, 615-620

25. Jiang JG, Chen CL, Card JW et al (2005) Cytochrome P450 2J2 promotes the neoplastic phenotype of carcinoma cells and is up-regulated in human tumors. Cancer Res 65, 4707-4715

26. Zheng S, Chheda MG and Verhaak RG (2012) Studying a complex tumor: potential and pitfalls. Cancer J 18, 107-114

27. Lamfers $M$, Idema S, van Milligen F et al (2009) Homing properties of adipose-derived stem cells to intracerebral glioma and the effects of adenovirus infection. Cancer Lett 274, 78-87

28. Studeny M, Marini FC, Champlin RE, Zompetta C, Fidler IJ and Andreeff $M$ (2002) Bone marrow-derived mesenchymal stem cells as vehicles for interferon-beta delivery into tumors. Cancer Res 62, 3603-3608

29. Montecalvo A, Larregina AT, Shufesky WJ et al (2012) Mechanism of transfer of functional microRNAs between mouse dendritic cells via exosomes. Blood 119, 756-766

30. Lee HK, Finniss S, Cazacu S et al (2013) Mesenchymal stem cells deliver synthetic microRNA mimics to glioma cells and glioma stem cells and inhibit their cell migration and self-renewal. Oncotarget 4, 346-361

31. Lee Y, El Andaloussi S and Wood MJ (2012) Exosomes and microvesicles: exracellular vesicles for genetic information transfer and gene therapy. Hum Mol Genet 21, R125-134 